\title{
ChemComm
}

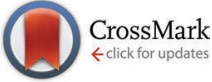

Cite this: Chem. Commun., 2015, 51, 3656

Received 3rd December 2014 Accepted 26th January 2015

DOI: $10.1039 / \mathrm{c} 4 \mathrm{cc} 09665 \mathrm{~g}$

www.rsc.org/chemcomm

\section{The synthesis and electrochemical characterization of bis(fluorosulfonyl)imide-based protic ionic liquids $\dagger$}

\author{
S. Menne, $\ddagger$ T. Vogl $\ddagger$ and A. Balducci*
}

\begin{abstract}
A protic ionic liquid containing the FSI anion has been synthesized for the first time and used as an electrolyte in an electrochemical storage device. This PIL-based electrolyte outperforms commonly used aprotic ionic liquids, maintaining the advantages and safety of ionic liquid-based electrolytes.
\end{abstract}

Lithium-ion batteries (LIBs) are presently considered as one of the most important electrochemical storage devices and in the last few years these devices have been proposed and introduced in a large number of new applications. ${ }^{1}$ In most of the new applications foreseen for LIBs, e.g. electric vehicles, safety represents a key aspect. Nevertheless, the commercially available LIBs contain electrolytes based on organic carbonates (e.g. ethylene carbonate (EC), diethyl carbonate (DEC)), which are highly flammable and volatile. ${ }^{2}$ In order to successfully introduce LIBs into the abovementioned new applications the introduction of new and safer electrolytes is needed.

Ionic liquids (ILs) are currently regarded as one of the most promising alternative electrolytes for LIBs. ${ }^{3}$ As a matter of fact, ILs display high thermal stability and extremely low volatility and it has been shown that the use of these alternative electrolytes might improve the safety of LIBs. ${ }^{4}$ Nevertheless, the performance of LIBs containing ILs as electrolytes is still not fully comparable with that of conventional devices. Particularly, the reduced lithium-ion transport typically observed in IL-based electrolytes represents a serious drawback, especially when high current densities are applied.

So far, basically all investigations concerning the use of IL as electrolytes for LIBs have been carried out using aprotic ionic liquids (AILs). Nevertheless, in a series of recent reports we showed that protic ionic liquids (PILs) represent a very promising alternative to AILs. ${ }^{5}$ PIL-based electrolytes for LIBs display all the

Westfälische Wilhelms-Universität Münster, Institute of Physical Chemistry, Corrensstr.28/30, 48149 Münster, Germany. E-mail: andrea.balducci@uni-muenster.de $\dagger$ Electronic supplementary information (ESI) available. See DOI: 10.1039/ c4cc09665g

¥ SM and TV equally contributed to this work. favourable properties of those based on AILs. ${ }^{6}$ However, thanks to the unique lithium environment present in the PIL, the use of this type of ILs makes possible the realization of LIBs with much higher performance at high current densities compared to that possible with AILs. ${ }^{7}$ Furthermore, since PILs are typically cheaper than AILs, the introduction of these innovative electrolytes could also be of importance for the development of safer and cheaper IL-based LIBs.

So far, only a very limited number of PILs have been used as electrolytes for LIB. These PILs contained pyrrolidinium and triethylammonium as cations, while bis(trifluoromethanesulfonyl)imide (TFSI) was used as the anion in most of the cases. ${ }^{5,7}$

It is well known that the selection of ions has a dramatic influence on the chemical-physical properties of ILs. ${ }^{8}$ Therefore, the synthesis and investigation of new types of PILs, having different anions and cations with respect to those so far investigated, appears to be of importance. As a matter of fact, such an investigation could give important indication about the ions, which should be preferentially selected for the synthesis of PILs designed for LIB applications.

In the past, it has been shown that the use of the anion bis(fluorosulfonyl)imide (FSI) is of particular interest in view of the realization of advanced IL-based electrolytes for LIBs. As a matter of fact, FSI-based ILs typically display lower viscosities (due to the smaller size of the anions) and higher conductivities than those containing TFSI. ${ }^{9}$

Taking this point into account, the investigation of FSI-based PILs appears therefore to be of great interest as it could provide important information in view of the realization of advanced LIBs containing ILs as electrolytes. To the best of our knowledge, however, there are no reports available about either the synthesis or the chemical-physical and electrochemical characterization of FSI-based PILs.

In this work we report for the first time about the synthesis, and chemical-physical and electrochemical characterization of an FSI-based PIL, $N$-butylpyrrolidinium bis(fluorosulfonyl)imide $\left(\mathrm{PYR}_{\mathrm{H} 4} \mathrm{FSI}\right)$. Initially, the synthesis of $\mathrm{PYR}_{\mathrm{H} 4} \mathrm{FSI}$ is described in detail. Afterward, the viscosity and conductivity of this PIL were 


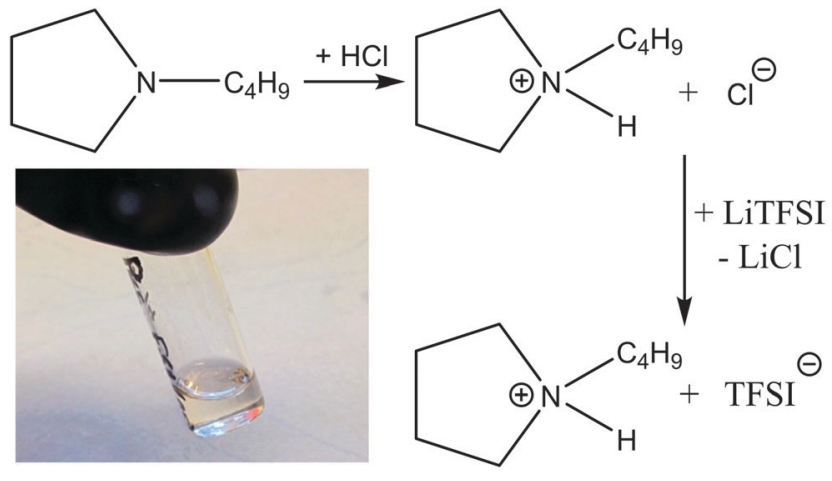

Fig. 1 Scheme of the synthesis.

evaluated and compared to that of the AIL $N$-butyl- $N$-methylpyrrolidinium bis(fluorosulfonyl)imide $\left(\mathrm{PYR}_{14} \mathrm{FSI}\right)$. Finally, both ILs were mixed with lithium bis(fluorosulfonyl)imide (LiFSI) to form electrolytes suitable for LIBs. The performance of devices containing these electrolytes was investigated and compared with that of systems using conventional electrolytes.

Fig. 1 schematizes the synthetic procedure used for the synthesis of $\mathrm{PYR}_{\mathrm{H} 4} \mathrm{FSI}$, which is similar to that previously described in ref. 10. The $\mathrm{PYR}_{\mathrm{H} 4} \mathrm{FSI}$ was characterized via NMR as follows: ${ }^{1} \mathrm{H}-\mathrm{NMR}$ $\left(\mathrm{CDCl}_{3}\right): \delta=0.94-0.98(\mathrm{t}, 3 \mathrm{H}, \mathrm{CH} 3) ; 1.37-1.46(\mathrm{~m}, 2 \mathrm{H}, \mathrm{CH} 2)$; 1.68-1.76 (m, 2H, CH2); 2.08-2.22 (m, 4H, CH2); 2.99-3.08 (m, 2H, CH2); 3.13-3.19 (m, 2H, CH2); 3.71-3.78 (m, 2H, CH2); $7.50(\mathrm{~s}, 1 \mathrm{H}, \mathrm{NH}) \mathrm{ppm} .{ }^{13} \mathrm{C}-\mathrm{NMR}\left(\mathrm{CDCl}_{3}\right): \delta=13.46 ; 19.68 ; 22.96$; 27.92; 55.09; $56.14 \mathrm{ppm} .{ }^{19} \mathrm{~F}-\mathrm{NMR}\left(\mathrm{CDCl}_{3}\right): \delta=53.18 \mathrm{ppm}$. (The NMR spectra of $\mathrm{PYR}_{\mathrm{H} 4} \mathrm{FSI}$ can be found in the ESI $\dagger$ ).

The water content of $\mathrm{PYR}_{\mathrm{H} 4} \mathrm{FSI}$ was found to be less than $10 \mathrm{ppm}$.

Fig. 2 compares the conductivity and the viscosity of PYR $_{\mathrm{H} 4} \mathrm{FSI}$ with those of the AIL PYR ${ }_{14} \mathrm{FSI}$ and the commercial electrolyte LP30 (containing $1 \mathrm{M} \mathrm{LiPF}_{6}$ in EC:DMC 1:1 w:w). As shown, the conductivity of both ILs is lower than that of LP30, but is similar over the entire investigated temperature range. This similarity in terms of conductivity between AILs and dry PILs has been already observed in ILs containing the TFSI anion, ${ }^{7}$ and it is therefore not unexpected. At $30{ }^{\circ} \mathrm{C}, \mathrm{PYR}_{\mathrm{H} 4} \mathrm{FSI}$ displays a conductivity of $7.4 \mathrm{mS} \mathrm{cm} \mathrm{cm}^{-1}$. This value is significantly higher than those observed for dry PIL having the TFSI anion (e.g. $\mathrm{PYR}_{\mathrm{H} 4}$ TFSI, which displays a conductivity of $4.0 \mathrm{mS} \mathrm{cm}^{-1}$ ). Furthermore, it is also important to notice that this value of conductivity is among the highest so far reported for dry PILs. As shown in Fig. 2B, the viscosity of the two ILs is considerably higher than that of LP30. However, in contrast to the conductivity, the viscosity of the two considered ILs is very different, and over the whole temperature range the viscosity of $\mathrm{PYR}_{14} \mathrm{FSI}$ is higher than that of $\mathrm{PYR}_{\mathrm{H} 4} \mathrm{FSI}$. At room temperature the difference between these two ILs is quite marked, and the viscosity of the PIL is more than $38 \%$ lower than that of the AIL. When the temperature is increased, the difference between the two ILs becomes smaller. The lower viscosity of $\mathrm{PYR}_{\mathrm{H} 4} \mathrm{FSI}$ is most likely due to the smaller size of the cation forming this IL compared to that of $\mathrm{PYR}_{14} \mathrm{FSI}$. Furthermore, the ion-ion interaction taking place in these two ILs is also likely to be different, leading to a lower viscosity for the PILs. ${ }^{11}$ As for the conductivity, it is important to remark that the viscosity of $\mathrm{PYR}_{\mathrm{H} 4} \mathrm{FSI}$ is significantly (almost 3 times) lower than that of dry PIL having the TFSI anions. Taking into account the values of conductivity and viscosity of $\mathrm{PYR}_{\mathrm{H} 4} \mathrm{FSI}$, in the case of PIL, as also for the AIL, the use of the FSI anion appears to be favourable for the design of IL with high conductivity and low viscosity.

In order to realize an electrolytic solution suitable for use in LIBs, PYR ${ }_{\mathrm{H} 4} \mathrm{FSI}$ was mixed with the lithium salt LiFSI $(1 \mathrm{M})$. With the aim of further investigating the difference between AIL and PIL-based electrolytes, a solution of $1 \mathrm{M}$ LiFSI in $\mathrm{PYR}_{14} \mathrm{FSI}$ was also prepared.

Table 1 reports a comparison of the cathodic and anodic limits, as well as of the overall electrochemical stability window (ESW) of the investigated electrolytes. As shown, while the anodic stability of the three electrolytes is comparable, the cathodic stability limit of the PIL-based electrolyte is $2 \mathrm{~V}$ smaller than that of the AIL and LP30. Consequently, the ESW of the PIL-based electrolyte is $2 \mathrm{~V}$ lower that that of the other electrolyte. The lower overall ESW of PIL-based electrolytes compared to that AIL-based electrolytes and organic electrolytes has been already reported in literature. ${ }^{12,13}$ Therefore, such a result is not surprising. Nevertheless, it is
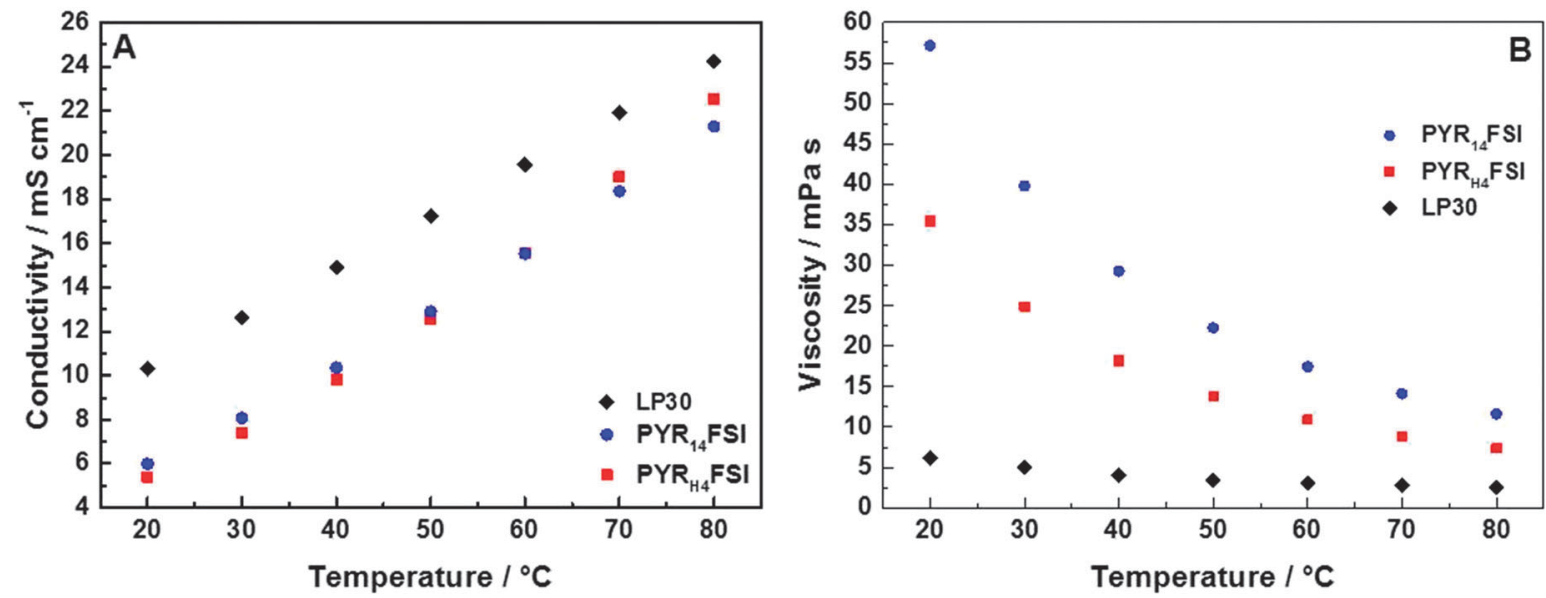

Fig. 2 Conductivity (A) and viscosity (B) of $\mathrm{PYR}_{\mathrm{H}_{4}} \mathrm{FSI}, \mathrm{PYR}_{14} \mathrm{FSI}$ and LP30. 
Table 1 Comparison of the electrochemical stabilities of the investigated electrolytes at $30{ }^{\circ} \mathrm{C}$ (working electrode Pt, scan rate: $1 \mathrm{mV} \mathrm{s}^{-1}$; all values refer to $\mathrm{Ag}$ reference)

\begin{tabular}{llll}
\hline Electrolyte & $\begin{array}{l}\text { Cathodic } \\
\text { limit }\end{array}$ & $\begin{array}{l}\text { Anodic } \\
\text { limit }\end{array}$ & $\begin{array}{l}\text { Overall } \\
\text { stability }\end{array}$ \\
\hline LP30 & $-3.0 \mathrm{~V}$ & $2.0 \mathrm{~V}$ & $5.0 \mathrm{~V}$ \\
$1 \mathrm{M} \mathrm{LiFSI}$ in PYR $_{14} \mathrm{FSI}$ & $-3.0 \mathrm{~V}$ & $2.0 \mathrm{~V}$ & $5.0 \mathrm{~V}$ \\
$1 \mathrm{M} \mathrm{LiFSI}$ in $\mathrm{PyR}_{\mathrm{H}} \mathrm{FSI}$ & $-1.0 \mathrm{~V}$ & $2.0 \mathrm{~V}$ & $3.0 \mathrm{~V}$ \\
\hline
\end{tabular}

important to remark that it has been shown that PIL-based electrolytes can also be successfully used in combination with carbonaceous anodes for LIB, e.g. graphite and soft carbon, and that the performances of PIL-based systems are never lower than those of AIL-based one. ${ }^{13}$ Thus, taking these results into account, the reduced ESW of PIL does not seem to hinder the compatibility of PIL-based electrolytes for battery materials.

The electrolytes investigated were used for the realization of a lithium-ion system containing a lithium iron phosphate (LFP)-based positive electrode and an activated carbon (AC)-based negative electrode (all details about electrode realization, composition and testing are provided in the ESI $\dagger$ ). The test with LP30 was carried out with the aim of evaluating the performance difference between systems containing conventional electrolytes and IL-based electrolytes.

Fig. 3A compares the discharge capacities (from 1C and 10C) of LFP-electrodes observed in three types of electrolytes mentioned above. As shown in the figure, the LFP electrode cycled in the conventional electrolyte LP30 displays the highest capacity at all considered C-rates. Nevertheless, it is very interesting to notice that the performances of the LFP electrodes cycled in the electrolyte containing $1 \mathrm{M}$ LiFSI in $\mathrm{PYR}_{\mathrm{H} 4} \mathrm{FSI}$ are not significantly lower than those of the conventional system. As shown, at 1C the capacity of the LFP electrode is comparable in these two electrolytes. Also when the C-rate is increased the LFP capacity observed in $1 \mathrm{M} \mathrm{LiFSI} \mathrm{in}$
$\mathrm{PYR}_{\mathrm{H} 4} \mathrm{FSI}$ is not dramatically lower. At 10C the LFP electrode cycled in $1 \mathrm{M}$ LiFSI in PYR ${ }_{\mathrm{H} 4} \mathrm{FSI}$ displays a capacity of $c a .100 \mathrm{~mA} \mathrm{~h} \mathrm{~g}^{-1}$, which is only $20 \%$ lower than that observed in the conventional electrolyte. As shown in the figure, when the $1 \mathrm{M}$ LiFSI in PYR ${ }_{14} \mathrm{FSI}$ was used the performance of the LFP electrode was significantly lower. At 1C the LFP displays a capacity of $c a .125 \mathrm{~mA} \mathrm{~h} \mathrm{~g}^{-1}$ in this electrolyte. This value is about $15 \%$ lower than those observed in the conventional and in the PIL-based electrolytes. When the C-rate is increased, this difference becomes much bigger. As a matter of fact, at 10C the LFP electrode displays a capacity of about $70 \mathrm{~mA} \mathrm{~h} \mathrm{~g}^{-1}$ in the AIL-based electrolyte. This value is $40 \%$ and $30 \%$ lower than those observed in LP30 and $1 \mathrm{M}$ LiFSI in $\mathrm{PYR}_{\mathrm{H} 4} \mathrm{FSI}$, respectively. It is important to notice that $1 \mathrm{M} \mathrm{LiFSI}$ in PYR ${ }_{14} \mathrm{FSI}$ is presently considered as one of the most suitable AIL-based electrolytes for high power applications. From the results reported above, it is evident that the use of PIL-based electrolytes is clearly more advantageous for the realization of high performance systems compared to the use of AIL-based electrolytes on LIBs. Fig. 3B compares the potential profiles (for the charge and the discharge) of the LFP electrode in the three considered electrolytes at the corresponding C-rates. In all three electrolytes the typical potential profiles of LFP are visible and a high efficiency can be observed from the charge and discharge curves at all C-rates. The overpotential difference between the charge and discharge plateaus increases from the organic to the PIL-based to AIL-based electrolyte and in each electrolyte with increasing C-rate, as expected. This observation is in line with the resulting capacities and the different viscosities.

After these tests, the cycling stability in the three electrolytes was also investigated. Fig. 4 shows the evolution of the LFP discharge capacity (over 50 cycles), as obtained during the charge-discharge test carried out at 1C. As shown, the systems cycled in $1 \mathrm{M}$ LiFSI in $\mathrm{PYR}_{\mathrm{H} 4} \mathrm{FSI}$ and LP30 display a very comparable capacity of more than $135 \mathrm{~mA} \mathrm{~h} \mathrm{~g}^{-1}$, stable over
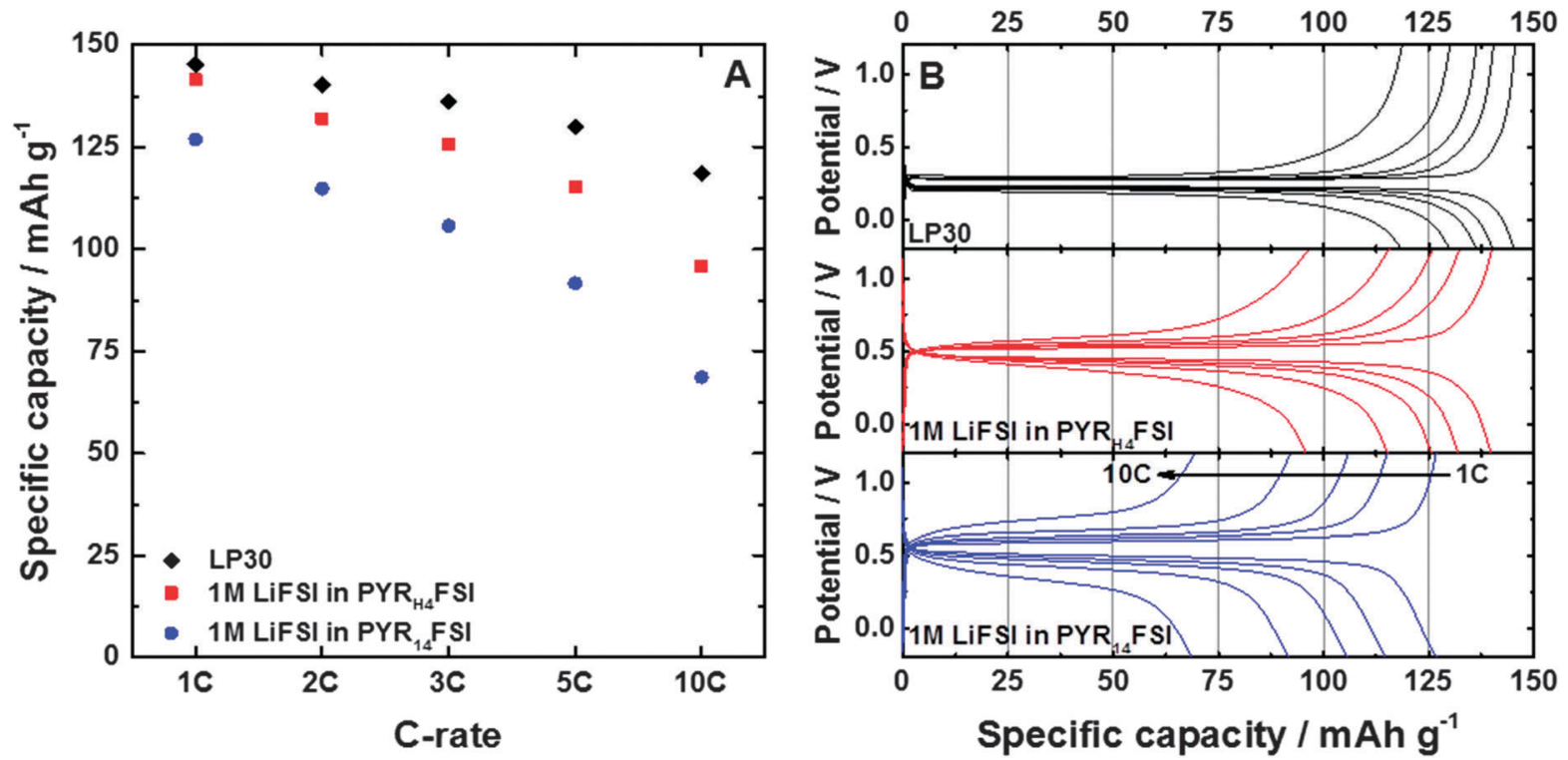

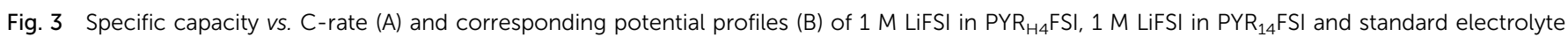
LP30. A silver wire was used as pseudo-reference and an oversized activated carbon-based electrode as counter electrode. 


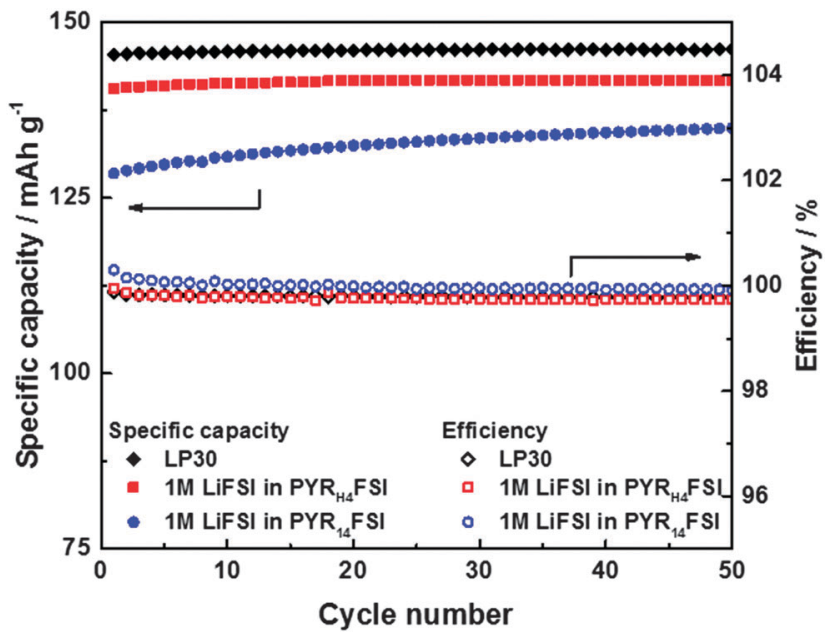

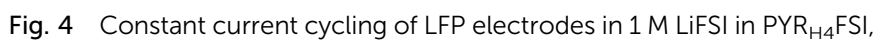
$1 \mathrm{M} \mathrm{LiFSI}$ in $\mathrm{PYR}_{14} \mathrm{FSI}$ and standard electrolyte LP30. A silver wire was used as pseudo-reference.

50 cycles. When the system was cycled in $1 \mathrm{M}$ LiFSI in PYR $_{14} \mathrm{FSI}$, the LFP electrode displayed an initial capacity of $125 \mathrm{~mA} \mathrm{~h} \mathrm{~g}{ }^{-1}$. During the cycling the capacity of the electrode increased and after 50 cycles reached a value of $c a .130 \mathrm{~mA} \mathrm{~h} \mathrm{~g}^{-1}$. Such an increase of capacity has been already observed in AIL-based electrolytes and it is originated due to electrode wetting, which increases over cycles. Interestingly, such an increase is not observed in the PIL-based electrolytes. As shown in the figure, the coulombic efficiency of the charge-discharge process was very high (close to $100 \%$ ) in all electrolytes, indicating that all of them are suitable for the realization of devices with stable performance.

In conclusion, the results of this investigation showed, for the first time, that FSI-based PILs, e.g. $\mathrm{PYR}_{\mathrm{H} 4} \mathrm{FSI}$, are very promising electrolytes for LIB. The synthesis of $\mathrm{PYR}_{\mathrm{H} 4} \mathrm{FSI}$ is a rather simple process, which leads to the realization of very pure ILs. When used dry, $\mathrm{PYR}_{\mathrm{H} 4} \mathrm{FSI}$ displays high conductivity and low viscosity at room and higher temperatures. We showed that using electrolytes containing this PIL it is possible to realize devices with significantly higher performance than those containing classical aprotic ionic liquids, e.g. PYR ${ }_{14}$ FSI. Although the performance observed in $1 \mathrm{M}$ LiFSI in PYR $_{\mathrm{H} 4} \mathrm{FSI}$ does not fully match those achievable in conventional electrolytes, the use of FSI-based PIL appears to be extremely appealing for the realization of high performance ILbased LIBs. Further investigations into these PILs are certainly necessary. Nevertheless, the results of this study can be seen as a significant step towards the realization of high performance ILbased systems, able to fill the performance gap existing between these innovative electrolytes and the conventional ones.

We wish to thank the University of Münster and the Ministry of Innovation, Science and Research of North Rhine-Westphalia (MIWF) within the project "Superkondensatoren und LithiumIonen-Hybrid-Superkondensatoren auf der Basis ionischer Flüssigkeiten" and the Bundesministerium für Bildung and Forschung (BMBF) within the project IES (contract number 03EK3010) for the financial support.

\section{Notes and references}

1 B. Scrosati and J. Garche, J. Power Sources, 2010, 195, 2419; N. Nitta, F. Wu, J. T. Lee and G. Yushin, Mater. Today, 2014, DOI: 10.1016/ j.mattod.2014.10.040.

2 K. Xu, Chem. Rev., 2004, 104, 4303.

3 D. R. MacFarlane, N. Tachikawa, M. Forsyth, J. M. Pringle, P. C. Howlett, G. D. Elliott, J. H. Davis, M. Watanabe, P. Simon and C. A. Angell, Energy Environ. Sci., 2014, 7, 232.

4 M. Galinski, M. Galiński, A. Lewandowski and I. Stpniak, Electrochim. Acta, 2006, 51, 5567; M. Armand, F. Endres, D. R. MacFarlane, H. Ohno and B. Scrosati, Nat. Mater., 2009, 8, 621.

5 S. Menne, J. Pires, M. Anouti and A. Balducci, Electrochem. Commun., 2013, 31, 39; N. Böckenfeld, M. Willeke, J. Pires, M. Anouti and A. Balducci, J. Electrochem. Soc., 2013, 160, A1.

6 A. Fernicola, B. Scrosati and H. Ohno, Ionics, 2006, 12, 95; C. A. Angell, Y. Ansari and Z. Zhao, Faraday Discuss., 2012, 154, 9; C. A. Angell, N. Byrne and J.-P. Belieres, Acc. Chem. Res., 2007, 40, 1228.

7 T. Vogl, S. Menne, R.-S. Kühnel and A. Balducci, J. Mater. Chem. A, 2014, $2,8258$.

8 J. F. Wishart, Energy Environ. Sci., 2009, 2, 956; M. Galinski, A. Lewandowski and I. Stepniak, Electrochim. Acta, 2006, 51, 5567.

9 A. Lewandowski, A. Hollenkamp, S. Donne and A. Best, J. Power Sources, 2010, 195, 2029; A. S. Best, A. I. Bhatt and A. F. Hollenkamp, J. Electrochem. Soc., 2010, 157, A903; E. E. Paillard, Q. Zhou, W. Henderson, G. B. Appetecchi, M. Montanino and S. Passerini, ECS Trans., 2009, 16, 51; I. A. Shkrob, T. W. Marin, Y. Zhu and D. P. Abraham, J. Phys. Chem. C, 2014, 118, 19661.

10 L. Timperman, P. Skowron, A. Boisset, H. Galiano, D. Lemordant, E. Frackowiak, F. Beguin and M. Anouti, Phys. Chem. Chem. Phys., 2012, 14, 8199.

11 S. Menne, T. Vogl and A. Balducci, Phys. Chem. Chem. Phys., 2014, 16, 5485.

12 G. B. Appetecchi, M. Montanino, A. Balducci, S. F. Lux, M. Winterb and S. Passerini, J. Power Sources, 2009, 192, 599.

13 S. Menne, M. Schroeder, T. Vogl and A. Balducci, J. Power Sources, 2014, 266, 208. 